\title{
CORRECTION
}

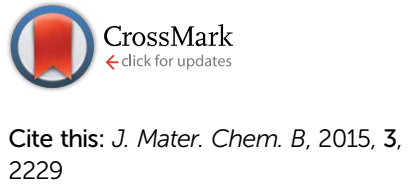

DOI: $10.1039 / c 5 t b 90031 j$

www.rsc.org/MaterialsB

\section{Correction: Tailor-made gemcitabine prodrug nanoparticles from well-defined drug-polymer amphiphiles prepared by controlled living radical polymerization for cancer chemotherapy}

\author{
Weiwei Wang, ${ }^{a}$ Chen Li, ${ }^{a}$ Ju Zhang, ${ }^{a}$ Anjie Dong ${ }^{b}$ and Deling Kong*a \\ Correction for 'Tailor-made gemcitabine prodrug nanoparticles from well-defined drug-polymer \\ amphiphiles prepared by controlled living radical polymerization for cancer chemotherapy' by Weiwei \\ Wang et al., J. Mater. Chem. B, 2014, 2, 1891-1901.
}

The authors would like to acknowledge and apologise for using some text from references 1 and 2 (below) without appropriate attribution in some sentences from sections 3.1 and 3.6 and that Scheme 1 was redrawn from Scheme 1 in reference 2 without appropriate attribution. The authors would like to clarify that this work is not the first example of such an amphiphilic polymer and related work is cited in the manuscript.

The in vivo drug concentration dose is incorrectly stated as $26 \mathrm{mg} \mathrm{kg}^{-1}$ throughout the manuscript (Abstract, sentence 8; section 2.8, paragraph 2, sentence 5; section 3.6, sentence 2; Discussion, paragraph 7, sentence 9). The correct dose administered was $0.693 \mathrm{mg} \mathrm{mL}{ }^{-1}$ obtained by concentrating the nanoparticle suspensions and the injection volume was $250 \mu \mathrm{L}$.

In several instances (section 3.1, paragraph 2, sentence 8; section 3.2, sentence 5; Table 1, column 7) the drug loading should be $30.4 \%$ and $17.7 \%$ for PMMA5 and PMMA11.2, rather than $43.7 \%$ and $21.5 \%$, respectively. The drug loading in the manuscript had been calculated using the formula Gem/PMMA, rather than by the more accurate formula Gem/(Gem + PMMA).

The conclusions remain unchanged.

\section{References}

1 D. Trung Bui, A. Maksimenko, D. Desmaële, S. Harrisson, C. Vauthier, P. Couvreur and J. Nicolas, Biomacromolecules, 2013, 14, 2837-2847.

2 S. Harrisson, J. Nicolas, A. Maksimenko, D. T. Bui, J. Mougin and P. Couvreur, Angew. Chem., Int. Ed., 2013, 52, 1678-1682.

The Royal Society of Chemistry apologises for these errors and any consequent inconvenience to authors and readers. 